\section{JTI}

JOURNAL OF

TRAUMA AND INJURY

\title{
Bilateral Chylothorax Due to Blunt Spine Hyperextension Injury: A Case Report
}

Hohyoung Lee, M.D., Sung Ho Han, M.D., Min Koo Lee, M.D., Ph.D., Oh Sang Kwon, M.D., Kyoung Hwan Kim, M.D., Jung Suk Kim, M.D., Soon-Ho Chon, M.D., Ph.D., Sung Ho Shinn, M.D., Ph.D.

Departments of Trauma Surgery and Thoracic and Cardiovascular Surgery, Cheju Halla Hospital, Jeju, Korea

Received: November 14, 2018

Revised: January 7, 2019

Accepted: January 19, 2019

\section{Correspondence to}

Soon-Ho Chon, M.D., Ph.D.

Departments of Trauma Surgery and Thoracic and Cardiovascular Surgery,

Cheju Halla Hospital, 65 Doryeong-ro, Jeju 63127, Korea

Tel: $+82-64-740-5039$

Fax: $+82-64-743-3110$

E-mail:sh.chon@hotmail.com
Bilateral chylothorax due to blunt trauma is extremely rare. We report a 74-year-old patient that developed delayed bilateral chylothorax after falling off a ladder. The patient had a simple 12th rib fracture and T12 lamina fracture. All other findings seemed normal. He was sent home and on the 5th day visited our emergency center at Halla Hospital with symptoms of dyspnea and lower back pain. Computer tomography of his chest presented massive fluid collection in his right pleural cavity and moderate amounts in his left pleural cavity with 12th rib fracture and T11-12 intervertebral space widening with bilateral facet fractures. Chest tubes were placed bilaterally and chylothorax through both chest tubes was discovered. Conservative treatment for 2 weeks failed, and thus, thoracic duct ligation was done by video assisted thoracoscopic surgery. Thoracic duct embolization was not an option. Postoperatively, the patient is now doing well and happy with the results. Early surgical treatment must be considered in the old patient, whom large amounts of chylothorax are present.

Keywords: Chylothorax; Blunt injury; Video assisted thoracoscopic surgery

\section{INTRODUCTION}

Minimally invasive thoracoscopic surgery has provided an attractive option for many radical procedures. Thoracosopic surgery is evolving and becoming more tangible. Chylothorax due to blunt trauma is seen in only 0.2 to $3 \%$ of all non-iatrogenic causes [1]. We report a case of bilateral chylothorax occurring in a 74-year-old patient, whom had thoracic duct ligated by thoracoscopic surgery. The patient was discharged on the (http://creativecommons.org/licenses/by-nc/4.0/) which permits unrestricted noncommercial use, distribution, and reproduction in any medium, provided the original work is properly cited. 
postoperative 12th day without recurrence and is happy with the results.

\section{CASE REPORT}

A 74-year-old male had visited a local clinic with symptoms of lower back pain. He was originally found with no significant injury and after simple X-rays of his chest and spine, he was sent home with pain killers. Five days later, he visited our emergency center at Halla Hospital with symptoms of dyspnea and lower back pain. Initial chest $\mathrm{X}$-rays presented massive amounts of fluid collection in his right pleural cavity (Fig. 1). Computer tomogram of his chest presented bilateral fluid accumulation (Fig. 2A) and presence of large osteophytes in his T11 and T12 spines (Fig. 2B). An emergent chest tube insertion was done and 3 liters of milky fluid was drained. Laboratory values of the fluid presented a triglyceride content of $1,554 \mathrm{mg} / \mathrm{dL}$ confirming chylothorax. Hyponatremia of $133 \mathrm{mEq} / \mathrm{L}$, Hypocalcemia of $7.5 \mathrm{mg} / \mathrm{dL}$ and low albumin level of 2.2 was seen at initial presentation. He was treated by fasting and total parenteral nutrition. Magnetic resonance imaging of his spine was taken two days later showing T11-12

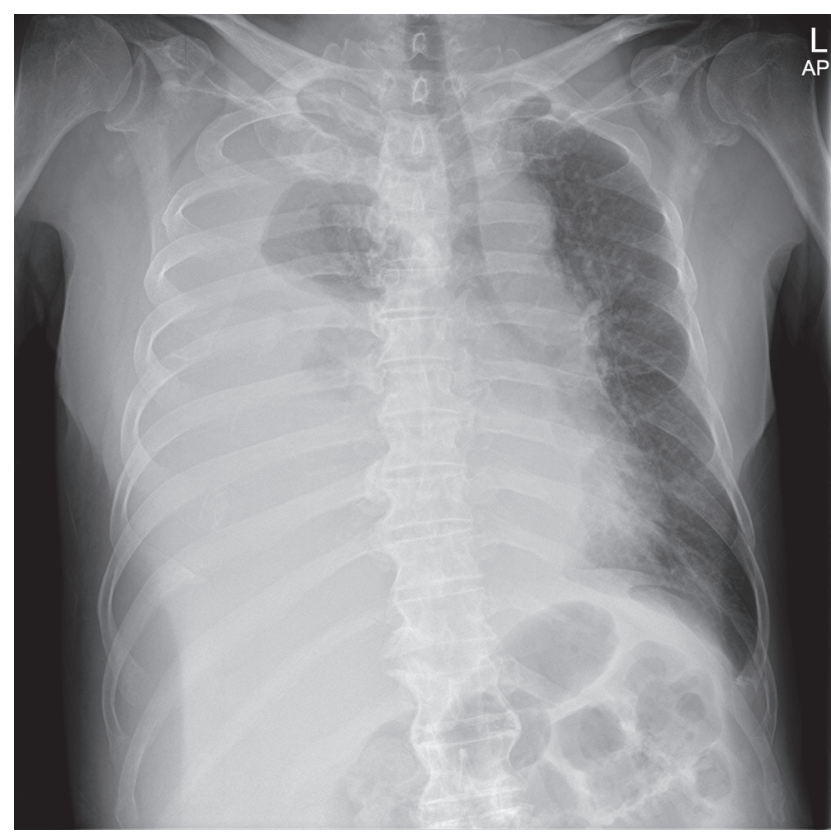

Fig. 1. Initial simple chest $\mathrm{X}$-ray showing large amount of right pleural fluid causing tension chylothorax. anterior longitudinal ligament rupture with anterior disc space widening and bilateral facet fractures. These findings were compatible with hyperextension injury. Three days later, a chest tube insertion was done on his left side due to increase in chylothorax. Low fat diet was attempted a week later, only to find recurring chylothorax. He was restarted on total parenteral nutrition. Three weeks after his injury or 2 weeks after admission, low fat diet was restarted to see if chylothorax would recur. Chylothorax persisted and lab values of the fluid presented triglyceride levels of $184 \mathrm{mg} / \mathrm{dL}$, cholesterol levels at $54 \mathrm{mg} / \mathrm{dL}$. He was draining chylothorax at about $900 \mathrm{~mL}$ per day. Diagnostic and interventional lymphangiograms were not available at Halla Hospital and thus, recommendation for transfer was done. The patient and guardians refused transfer and thus a thoracic duct ligation by video assisted thoracoscopic surgery was scheduled.

The procedure was done by general anesthesia with a double lumen endotracheal tube. Three ports were utilized. The leak was seen laterally to his spine near to his superior costal facet at the level of his twelfth vertebra. Total rupture at duct was suspected. After failure to identify the site of leakage, we decided to go deep into his retroperitoneal space below his diaphragm. Dissection was done below his diaphragm level and the tissue between his vertebra and his aorta en toto was ligated with 3-0 vicryl suture and then with three $5 \mathrm{~mm}$ endoclips (Fig. 3). Cessation of chyle leak was confirmed. Talc flurry at the site was sprayed after irrigation and then insertion of a $28 \mathrm{Fr}$. chest tube was done.
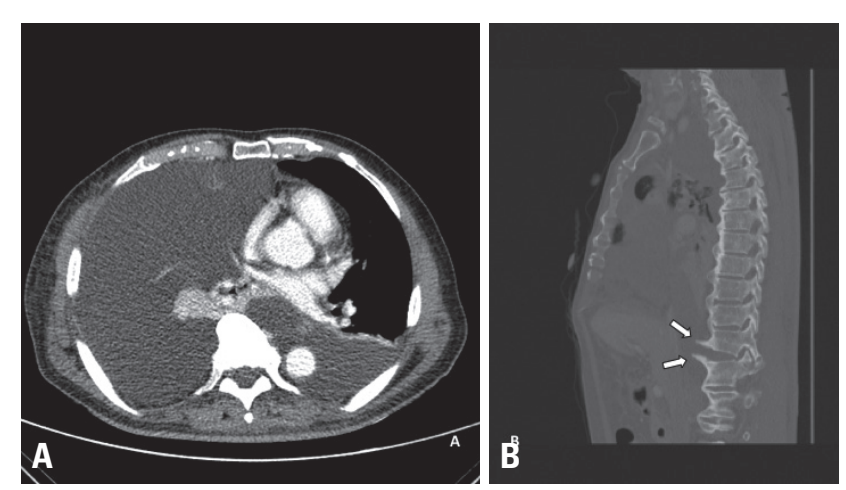

Fig. 2. Chest computer tomogram showing bilateral chylothorax (A) and huge osteophytes (arrows) in his T11-12 with hyperextension injury among a background of diffuse idiopathic skeletal hyperostosis (B). 
A small amount of chylothorax was seen initially, possibly due to remaining chylothorax in his pleural cavity. On postoperative 1st day, the chylothorax completely stopped. His chest tube was removed on postoperative fifth day and he was discharged on his postoperative 12th day at the request of his guardians. He was put on a thoracolumbosacral orthosis without surgery. He is free from his chylothorax now 2 months after the procedure as evidenced by his postoperative 5 week chest $\mathrm{X}$-ray, is free from pain, and is happy with the results (Fig. 4).

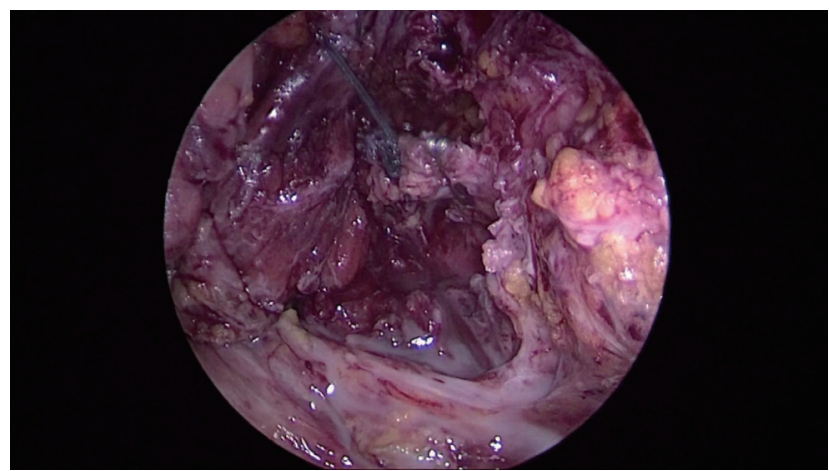

Fig. 3. Video capture presenting the ligated and clipped thoracic duct after dissection below his diaphragm within retroperitoneal space.

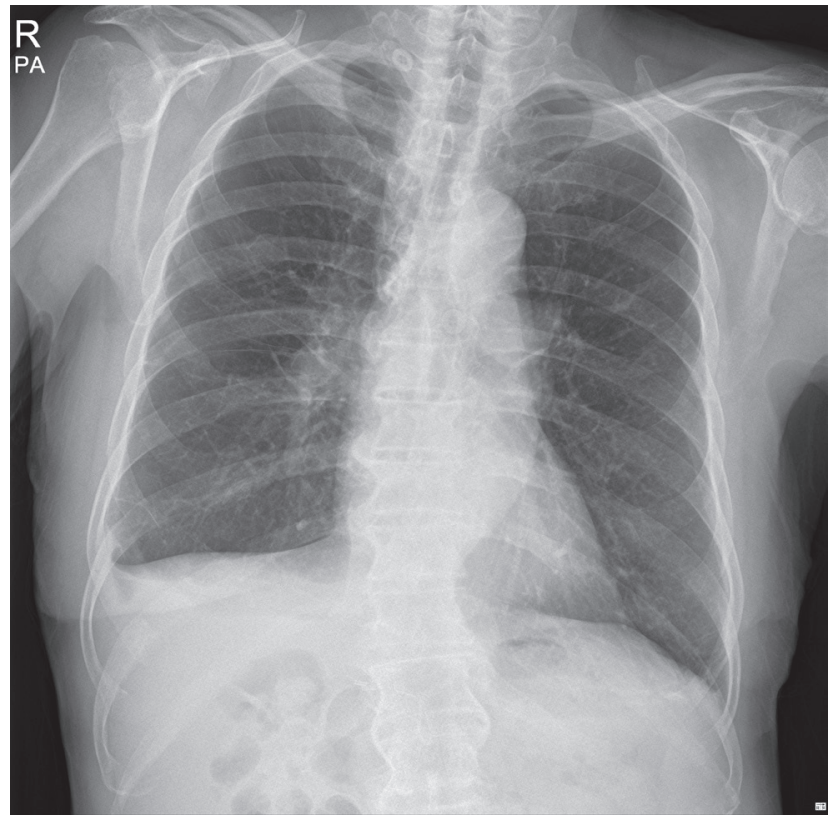

Fig. 4. Simple chest $X$-ray showing complete resolution 5 weeks after thoracic duct ligation.

\section{DISCUSSION}

The thoracic duct was first discovered by the Jean Pecquet in 1651 [1]. Flow rates through the duct reach $100 \mathrm{~mL} / \mathrm{kg}$ of body weight. That is about $2.5 \mathrm{~L}$ per day. Sixty to $70 \%$ of ingested fat, including vitamins, is absorbed through the thoracic duct. The peak levels of chylomicrons are about 3 hours postprandially [1]. Chylothorax caused by non-iatrogenic trauma is quite rare. Blunt trauma causes about 0.2 to $3 \%$ of all chylothorax and about 0.9 to $1.3 \%$ in penetrating trauma. The first case of traumatic duct injury was noted by Quincke in 1875 [1]. Non-iatrogenic causes range from penetrating trauma by stab or gunshot wounds, blunt trauma, thoracic spine fracture, posterior rib fracture and even coughing or sneezing [1]. Injury above the 5th and 6th thoracic vertebrae results in a left sided chylothorax and those below usually result in right sided chylothorax. The most common mechanism of injury is sudden hyperextension of the spine, resulting in rupture of the duct or by shearing of the crus of the diaphragm. Other mechanisms include direct injury due to vertebral fracture and injury due to penetration of the duct by osteophytes/exostoses [2]. In our particular case, a combination of hyperextension injury and tear caused by osteophytes seem the reason for bilateral chylothorax. A triglyceride level of over $110 \mathrm{mg} / \mathrm{dL}$ is considered diagnostic. Levels below $50 \mathrm{mg} / \mathrm{dL}$ can basically rule out chy lothorax in $86 \%$, however levels between 50 and $110 \mathrm{mg} / \mathrm{dL}$ need differential diagnosis [3]. The $14 \%$ below $50 \mathrm{mg} / \mathrm{dL}$ can be confirmed by the presence of chylomicrons in the fluid. Clearing of the fluid with addition of ethylether helps diagnose pseudochylothorax. Pseudochylothorax can be caused by longstanding pleural effusion and can be confused with true chylothorax.

Typical clinical manifestations include dyspnea and chest pain. Delayed chylothorax in blunt trauma may occur between 2 to 7 days after injury [3]. Our particular case took 5 days to occur. The most common electrolyte imbalances are hyponatremia and hypocalcemia. Acidosis at $\mathrm{pH}$ levels of 7.4 to 7.8 is also a common finding [3]. This was also seen in our case. Bioavailability of amiodarone and digoxin are lost in chyle, thus, this must be kept in consideration when these drugs are administered. Loss of proteins, immunoglobulins, $\mathrm{B}$ and $\mathrm{T}-$ 
lymphocytes can lead to immunologic compromise [2]. Lymphocyte predominance of $>1,000$ cells/ $\mu \mathrm{L}$, fluid to serum cholesterol ratios of less than 1 , and triglyceride ratio of greater than 1 can help with diagnosis.

Recently, thoracic duct embolization provides an attractive option. Embolization can be achieved with the use of n-butyl cyanoacrylate with success rates approaching $90 \%$ with a complication rate of $3 \%$ [1]. Leaks in small collaterals need to be done with such bioglues. Conservative methods succeed in only $50 \%$ of the cases of chylothorax caused by trauma. Conservative methods include fasting, total parenteral nutrition and low fat diets or medium chain triglycerides that are directly absorbed through the portal system. Octreotide can be administered subcutaneously or intravenously at 50 to 300 micrograms three times daily ( 2 to $68 \mathrm{mcg} / \mathrm{kg} /$ day). Surgical indications include [1] chyle leak greater than 1.5 liters per day, [2] drain greater than 1 liter per day for five or more days, [3] persistent flow for greater than 2 weeks, or [4] rapid nutritional deterioration $[3,4]$. Surgical duct ligation achieves success rates of $70 \%$ [3]. Thoracoscopic surgical methods also provide an attractive option. Endoclips and nonabsorbable sutures describe some of the armamentarium. Mortality rates can reach up to $50 \%$ without proper treatment and drop to as low as $10 \%$ with aggressive treatment [1].

Bilateral chylothorax after blunt trauma is a very rare event. Prompt diagnosis and early treatment is essential and may be life-saving. Although surgical indications have been established, early surgical intervention or interventional thoracic duct embolization must be considered in the elderly and severe trauma patient.

\section{REFERENCES}

1. Pillay TG, Singh B. A review of traumatic chylothorax. Injury 2016;47:545-50

2. Lee J, Cho JS, Hoseok I, Kim YD. Delayed right chylothorax after left blunt chest trauma: a case report. J Med Case Rep 2017;11:98.

3. Mohamed M, Alshenawy W, Kegarise C, Betten D. Bilateral chylothorax due to blunt trauma without radiographic evidence of traumatic injury. Clin Pract Cases Emerg Med 2017:1:111-4.

4. Idris K, Sebastian M, Hefny AF, Khan NH, Abu-Zidan FM. Blunt traumatic tension chylothorax: case report and minireview of the literature. World J Clin Cases 2016;4:380-4. 